\title{
A SHORT PROOF OF THE EQUIVALENCE OF KMP AND RNP IN BANACH LATTICES AND PREDUALS OF VON NEUMANN ALGEBRAS
}

\author{
V. CASELLES
}

(Communicated by William J. Davis)

\begin{abstract}
In this note we give a unified approach to the equivalence between the Krein-Milman property and the Radon-Nikodym property in Banach lattices and preduals of von Neumann algebras.
\end{abstract}

It is by now well known that, for a Banach lattice, the Krein-Milman property (abbreviated KMP) and the Radon-Nikodym property (RNP) are equivalent [2]. The proof given in [2] combines techniques of [1] and [4]. On the other hand, as remarked in [3], KMP and RNP are also equivalent for preduals of von Neumann algebras. It is our purpose in this note to give a short proof of both results using the main result of $[\mathbf{1}]$ and an easy argument. We prove a more general result, in terms of ordered Banach spaces, enhancing both results above. Let $E$ be an ordered Banach space [6] with a positive cone $E_{+}$. Then the bidual $E^{\prime \prime}$ of $E$ is also an ordered Banach space whose positive cone will be denoted by $E_{+}^{\prime \prime}$. We identify $E$ with the canonical image of $E$ into $E^{\prime \prime}$. We say that $E_{+}$is solid in $E_{+}^{\prime \prime}$ if, for every $z \in E_{+}^{\prime \prime}$ and every $x \in E$ such that $z \leq x$, we have that $z \in E_{+}$. We can now state our theorem.

THEOREM. Let $E$ be an ordered Banach space such that $E_{+}$is solid in $E_{+}^{\prime \prime}$. Let $C$ be a closed, bounded, convex subset of $E_{+}$with the KMP. Then $C$ has the RNP.

ProOF. If $C$ does not have the RNP, there exists a closed, convex subset $D$ of $C$ such that $D$ does not contain any extreme point of its $w^{*}$-closure $\tilde{D}$ in $E^{\prime \prime}$. Let $\operatorname{ex}(D), \operatorname{ex}(\tilde{D})$ denote the set of extreme points of $D$ and $\tilde{D}$, respectively. Let $z \in \operatorname{ex}(D)$. Since $z \notin \operatorname{ex}(\tilde{D})$, there exists $a, b>0, a+b=1$, and $z_{1}, z_{2} \in \tilde{D}$ such that $z=a z_{1}+b z_{2}$. If $z_{1} \in E$, then $b z_{2} \in E$. Since $b>0, z_{2} \in E$. Hence, $z_{1}, z_{2} \in$ $\tilde{D} \cap E=D$ and $z \notin \operatorname{ex}(D)$. This contradiction proves that $z_{1} \notin E$. Similarly, $z_{2} \notin E$. But $\tilde{D} \subseteq E_{+}^{\prime \prime}, z_{1} \leq a^{-1} z, z_{2} \leq b^{-1} z$ together with our assumption imply that $z_{1}, z_{2} \in E$. This contradiction shows that $\operatorname{ex}(D)=\varnothing$ and $C$ does not have the KMP.

A Banach lattice $E$ with the KMP does not contain a copy of $c_{0}$. Hence $E$ is weakly sequentially complete and $E_{+}$is solid in $E_{+}^{\prime \prime}[\mathbf{5}$, II.5]. Taking $C:=$ $\left\{x \in E_{+}:\|x\| \leq 1\right\}$, Theorem 1 proves that $C$ is a Radon-Nikodym set. Therefore, positive linear operators from $L^{1}[0,1]$ into $E$ are representable. Since every

Received by the editors December 24, 1986.

1980 Mathematics Subject Classification (1985 Revision). Primary 46B22; Secondary 46B30.

Key words and phrases. Ordered Banach space, solid set, Banach lattice, von Neumann algebra, Krein-Milman property, Radon-Nikodym property. 
bounded, linear operator $T: L^{1}[0,1] \rightarrow E$ is the difference of two positive operators [5, IV.1.5], we obtain

COROllaRY 1 [2]. If $E$ is a Banach lattice with the $K M P$, then $E$ has the $R N P$.

Let $M_{*}$ be the predual of a von Neumann algebra $M . M_{*}$ is an ordered Banach space whose positive cone $\left(M_{*}\right)_{+}$(= the set of all normal, positive linear functionals on $M\left[7\right.$, III.4] is solid in $\left(M^{\prime}\right)_{+}\left[\mathbf{7}\right.$, III.2.14]). If $M_{*}$ has the KMP, by Theorem 1, $C:=\left\{f \in\left(M_{*}\right)_{+}:\|f\| \leq 1\right\}$ has the RNP. Using a compactness argument and the fact that there exists a contractive projection $P$ from $M^{\prime}$ onto $M_{*}$ with $P\left(\left(M^{\prime}\right)_{+}\right) \subseteq$ $\left(M_{*}\right)_{+}$, one proves that all bounded, linear operators $T: L^{1}[0,1] \rightarrow M$ with $T$ (unit ball of $\left.L^{1}\right) \subseteq C-C$ can be written as $T=T_{1}-T_{2}$ where $T_{1}, T_{2}: L^{1}[0,1] \rightarrow M$ map the unit ball of $L^{1}[0,1]$ into $C$, hence are representable. Therefore, the real part of $M$ (= the set of normal hermitian functionals) has the RNP. Since any functional $f \in M_{*}$ can be written as $f=f_{1}+i f_{2}$ where $f_{1}=2^{-1}\left(f+f^{*}\right), f_{2}=(2 i)^{-1}\left(f-f^{*}\right)$, and $f^{*}(x):=\overline{f\left(x^{*}\right)}, x \in M$, it is a routine argument to show that $M_{*}$ has the RNP. We have obtained

COROLLARY 2 [3]. In the predual of a von Neuman algebra the KMP and the RNP are equivalent.

ACKNOWLEDGMENT. I gratefully acknowledge a grant from the Ministerio de Educacion y Ciencia de España. This paper was written when I was at the University of Tübingen during the academic year 1986-87. I would like to thank the AG Funktionalanalysis of this University and especially Professors H. H. Schaefer and R. Nagel for their hospitality. I am also indebted to Professor A. Marquina (University of Valencia) for his constant help and advice.

\section{REFERENCES}

1. J. Bourgain, A geometric characterization of the Radon-Nikodym property in Banach spaces, Compositio Math. 36 (1978), 3-6.

2. J. Bourgain and M. Talagrand, Dans un espace de Banach reticulé solide, la propriété de RadonNikodym et celle de Krein-Milman sont equivalentes, Proc. Amer. Math. Soc. 81 (1981), 93-96.

3. C. -H. Chu, A note on scattered $C^{*}$-algebras and the Radon-Nikodym property, J. London Math. Soc. (2) 24 (1981), 533-536.

4. N. Ghoussoub and M. Talagrand, Order dentability and the Radon-Nikodym property in Banach lattices, Math. Ann. 243 (1979), 217-225.

5. H. H. Schaefer, Banach lattices and positive operators, Springer-Verlag, Berlin and New York, 1974.

6. _ Topological vector spaces, Macmillan, 1966.

7. M. Takesaki, Theury of operator algebras. I, Springer-Verlag, Berlin and New York, 1979.

Facultad de Matematicas, C/Dr. Moliner, 50. Burjasot (Valencia), Spain 